\title{
Electrochemical techniques for photoelectrode characterisation
}

Franky E. Bedoya-Lora, Isaac Holmes-Gentle and Anna Hankin*

Affiliations:

Dr F. E. Bedoya-Lora

Centro de Investigación, Innovación y Desarrollo de Materiales - CIDEMAT, Universidad de Antioquia, Cr. 53 No 61 - 30, Torre 2, Lab. 330, Medellín, Colombia

Dr I. Holmes-Gentle

Laboratory of Renewable Energy Science and Engineering, Institute of Mechanical Engineering,

École Polytechnique Fédérale de Lausanne, 1015 Lausanne, Switzerland

Dr A. Hankin

Department of Chemical Engineering, Imperial College London, South Kensington Campus, SW7 2AZ, United Kingdom

*E-mail: anna.hankin@imperial.ac.uk 


\begin{abstract}
Photoelectrodes enable simultaneous light absorption and catalysis of water splitting reactions. Their performance is established using electrochemical characterisation methods. Besides basic characterisation techniques such as voltammetry and chronoamperometry, employed in the dark or under illumination, more advanced techniques including (photo-)electrochemical impedance spectroscopy, intensity-modulated impedance spectroscopy and transient absorption spectroscopy can be used to evaluate key parameters and processes. For some of these techniques, data is often interpreted using over-simplified models, leading to the calculation of unreliable parameters. The values of the flat band potential and charge transfer efficiency depend heavily on the methods used to determine them and it is recommended that the values are corroborated using multiple techniques. Lastly, certain 'efficiencies' defined in the literature for electrically biased systems should be revised.
\end{abstract}

\title{
Keywords
}

photoelectrode, solid - liquid interface, characterisation, solar fuels, photoelectrochemistry 


\section{Introduction}

Photoelectrodes are an integral part of photoelectrochemical systems and their properties directly impact the overall device performance. The purpose of a photoelectrode is to generate the necessary electrochemical potential through the absorption of photons and to either partially or fully support an oxidation and/or reduction process. The effectiveness of the overall process depends on the efficiencies of each step, sequentially: (i) light harvesting, (ii) electron-hole separation, (iii) charge transport and (iv) (photo-)catalysis. Losses in the space charge layer contribute to the so-called surface recombination losses, while bulk recombination occurs beyond the space charge layer. The effects of these recombination processes on the photocurrent are shown in Figure 1.

Photoelectrodes are synthesized with specific reactions in mind and so in addition to the constituent semiconductor band gap(s), which determine the theoretical solar to electrical energy conversion efficiencies, the conduction/valence band edges need to align favourably with the reversible potentials of the reduction/oxidation reaction, respectively. Thus far, the suitability of a given semiconductor can be estimated theoretically [1,2] but, ultimately, the performance of a photoelectrode has to be evaluated experimentally using (photo-)electrochemical techniques.
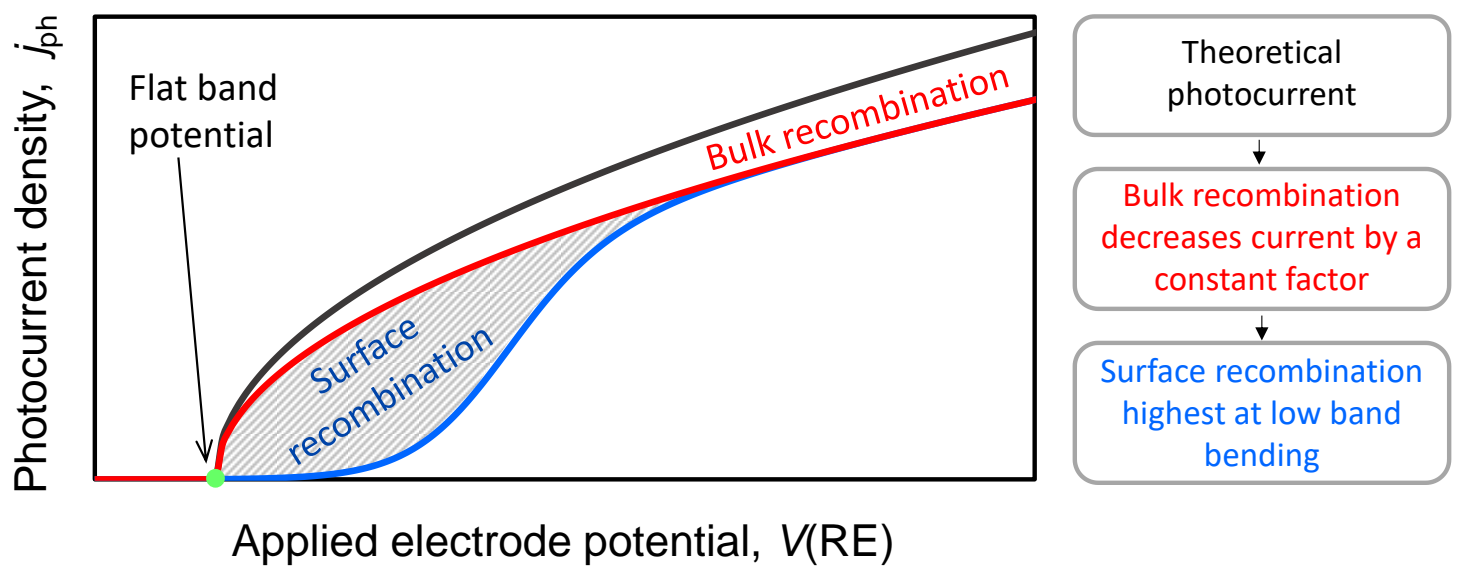

Figure 1. Schematic of anodic photocurrent densities as a function of applied potential: (a) predicted theoretically by the Gärtner-Butler equation (black), decreased by bulk recombination (red) and further by surface recombination (blue). 


\subsection{Basic characterisation}

The first tests on a photoelectrode are designed to answer the basic questions: (i) is the electrode photoactive, followed by: (ii) what is the photocurrent onset potential and (iii) what is the maximum photocurrent? These questions are answered by employing the photoelectrode as a working electrode in a three-electrode electrochemical cell, and comparing its performance as a function of applied potential in the dark and under illumination by white light $\left(100 \mathrm{~mW} \mathrm{~cm}^{-2}\right.$ with the spectral composition of the global AM 1.5 irradiance). An electric potential is applied to the photoelectrode against a reference electrode to simulate the realistic voltage range relevant to a complete photoelectrochemical device.

\subsubsection{Open circuit potential (OCP)}

OCP is the potential of the photoelectrode when it is in equilibrium with the electrolyte. The equilibration process usually entails surface charge redistribution, causing a degree of band bending in the semiconductors. Absorption of photons, and the consequent formation of electronhole pairs, compensates for this charge imbalance and causes the bands to unbend. Hence, an effective photoelectrode will exhibit different OCPs under dark and light conditions. If the OCP does not respond to light, the electrode is unlikely to display significant photoactivity under applied bias. OCP at high light intensities can also give an approximation of the position of fermi level at the flat band condition [3], though the measured potentials can be affected by fermi level pinning, as evidenced from Figure 2(a) and (b).

\subsubsection{Chopped photocurrent voltammetry}

This method determines photoelectrode performance under transient conditions. The measurements are conducted under potentiodynamic conditions and illumination is periodically switched on and off, or 'chopped'. It provides quick evidence of photoactivity, as well as the degree of biasing required for the photocurrent to plateau, as shown in Figure 2(c). The duration of the dark and light periods can vary and may or may not capture steady-state behaviour in addition to initial transient responses. 


\subsubsection{Steady state photocurrent and IPCE}

Transient photocurrents are typically higher than steady state photocurrents and are not representative of the photoelectrode output. Steady state photocurrents can be evaluated from voltammograms recorded at a scan rate of ca. $1 \mathrm{mV} \mathrm{s}^{-1}$, which is usually low enough for a near steady state response, or measured under potentiostatic conditions. Steady state photocurrents measured under illumination by monochromatic light are used to determine the incident photon to current conversion efficiencies (IPCE) according to Equation (1), over a set of applied potentials, as shown in Figure 2(d) [4]. The nomenclature can be found in Table 1.

$$
\operatorname{IPCE}_{\lambda}=\frac{j_{\mathrm{ph}, \lambda} \cdot h \cdot c}{P_{0, \lambda} \cdot e \cdot \lambda}
$$

IPCE is also valuable when the same material might be exposed to different light sources, for example when migrating experiments from artificial to natural light, as the performance of the electrode can be estimated by integration of the IPCE over the desired spectra. For IPCE measurements it is necessary to have access to monochromators or a wide range of LEDs with different wavelengths.

The addition of sacrificial reagents such as hydrogen peroxide [5], methanol [6] or hydrogen sulfide $[7,8]$ to the electrolyte facilitates charge transfer, allowing the estimation of bulk and surface recombination losses even under steady state conditions. However, some photoelectrodes exhibit current doubling effects rendering this approach unsuitable [9].

\subsubsection{Photoelectrode stability and long-term performance}

Stability of individual photoelectrodes is usually evaluated under potentiostatic conditions; decay in photocurrent over time signifies loss in performance. Factors that most often cause a decrease in current are (i) (photo-)corrosion, (ii) accumulation of by-products and (iii) increased coverage by bubbles, which can block the electroactive area and decrease the intensity of light reaching the surface [10-12]. The latter issue could be obviated by enhancing the convection (flow or stirring) near the electrode surface. Though some attempts for rigorous benchmarking have been proposed by the photoelectrochemical community, there is currently no concrete time period or experimental 
conditions over which stability data has to be provided in publications, either for a photoelectrode or the complete photoelectrochemical reactor [12,13]. Poor material stabilities remain a bottleneck, ranging from minutes to weeks, but are also being reported in an unsystematic manner and often

just described qualitatively. The lack of a standard protocol for stability tests needs to be addressed by the community.

Ultimately, as sunlight is intermittent, the stability of the photoelectrodes and reactors under multiple cycles of dark/light conditions would need to be evaluated to demonstrate potential for industrial deployment.

\subsection{Advanced characterisation}

In-situ (photo-)electrochemical characterisation techniques can be used to extract valuable parameters that characterise the semiconductor and its interface with the electrolyte and are important for enabling comparisons of material performance and elucidating the effects of material composition, nanostructuring as well as the electrolyte chemistry on the IPCE.

The typical parameters of interest are: the flat band potential, semiconductor dopant concentration and the potential-dependent interfacial charge transfer efficiency.

\subsubsection{Electrochemical impedance spectroscopy (EIS)}

EIS is an extremely useful and proven characterisation technique and, given its transient nature, it is possible to extract useful information about the kinetics and the positions of energy bands at the surface of the electrodes and evaluate all three parameters mentioned above. However, EIS can be highly sensitive to the conditions at the electrode | electrolyte interface such as doping levels, nanostructure, $\mathrm{pH}$, concentrations of redox species, and the data can be all too easily processed using invalid assumptions [14].

For example, the flat band potential is an important reference state relative to which photoelectrochemical kinetics may be assessed, but the assumptions made during flat band potential determination from interfacial capacitance measurements have led to an implausibly wide range of values being reported for the same material, as shown in Figure 2(a). The use of the Mott- 
Schottky Equation (2), written here for an n-type material, requires three key assumptions to be valid: (i) the applied potential is dropped across the semiconductor only - this is not valid for heavily doped semiconductors, which demonstrate quasi-metallic behaviour, (ii) $C_{\mathrm{SC}}$ is assumed to be the same as the interfacial capacitance - the contribution of the Helmholtz layer is neglected a priori, and (iii) the semiconductor surface is perfectly flat. When these assumptions are made erroneously, which is often, the consequences are an incorrect slope and intercept of the plot $[2,16]$.

$$
\frac{1}{C_{\mathrm{SC}}^{2}}=\frac{2}{\varepsilon_{0} \varepsilon_{r} e n}\left(V(\mathrm{RE})-V_{\mathrm{FB}}(\mathrm{RE})-\frac{k_{B} T}{e}\right)
$$

An example of a Mott-Schottky plot is shown in Figure 2(e). Equation (2) can also be used to estimate $\varepsilon_{\mathrm{r}}$ and $n$ but the catch is that to estimate one, the other must be known already.

Impedance spectroscopy under illumination, or PEIS, has been used to estimate kinetic rates of charge recombination and transfer processes, which in turn can be used to estimate interfacial charge transfer efficiencies $[7,8,15,16]$.

Several strategies have been proposed to analyse and interpret impedance spectra. Fitting to equivalent electrical circuits, as indicated in Figure 2(f), is the most used methodology where resistances and capacitances are used to calculate time constants of the processes involved. However, there is still some controversy surrounding the representation of charge flow pathways by the circuits and the physical meaning of some of these fitted parameters, especially when constant phase elements (CPE) are used as an alternative to pure capacitances [17]. Notwithstanding, the use of CPE is still a recommended practice coupled with the calculation of effective capacitances using appropriate mathematical formulations; the greater the deviation from ideal capacitance, the less reliable the calculated effective capacitances are [18,19]. Distribution of relaxation times (DRT) has also been proposed as an approach that does not require an a priori model to extract data from the impedance spectra and allows a direct estimation of time constants [20-22]. 


\subsubsection{Intensity modulated photocurrent spectroscopy (IMPS)}

IMPS can be used to extract kinetic rates and charge transfer efficiencies as a function of potential [23-25]. The use of this approach requires judicious analysis, accounting for non-linear behaviour between photocurrent and light intensity, incurring additional experimental procedures besides EIS [21]. However, IMPS is able to separate the recombination and charge transfer processes more effectively than PEIS. Fitting to equivalent electrical circuits and calculation of the distribution of relaxation times (DRT) can also be used to interpret IMPS data. Due to the atypical shape of the IMPS spectra, shown in Figure 2(g), the choice of electrical circuits are still a matter of debate [26]. Some researchers use circuits that yield better fittings regardless of the physical meaning of some of the fitted parameters, such as negative capacitances and the exponential term of CPEs, while others are in search of circuits that can imitate better the phenomenological processes shown in the IMPS spectra [24].

\subsubsection{Chopped photocurrent or transient photocurrent}

Transient photocurrent is probably the most used and expeditious technique of all, when implemented under potentiostatic conditions. It can be used to estimate peak photocurrents (when charge recombination is not the limiting process) and 'stable' photocurrents when all processes are at equilibrium. From that, kinetic rates and charge transfer efficiencies can be easily estimated $[27,28]$. Mechanical choppers are discouraged for transient photocurrent experiments, while fast response LEDs are preferred [21]. LEDs can replace Xe arc lamps as fast response systems $(<100$ ns), making this approach a very reproducible and convenient technique. Transient photocurrents are typically measured in the millisecond to second timescale.

More recently, chopped photocurrent, coupled to voltammetry at low scan rates $\left(<1 \mathrm{mV} \mathrm{s}^{-1}\right)$, has been used to estimate flat band potentials as the point where there is a transition between n-type and p-type behaviour of the semiconductor [14]. These measurements yield more reproducible results than the Mott-Schottky approach and give a narrower window of flat band potential values, as shown in Figure 2(a). 


\subsubsection{Transient absorption spectroscopy (TAS)}

TAS, as a pump-probe technique helps track the transient changes in the optical absorbance by using a pulsed laser excitation (pump) and a second light beam as probe [29,30]. It has been used to measure the half-life of charge carries in photoelectrodes in the femtosecond to millisecond timescale [31,32]. Moreover, quasi steady-state pump-probe spectroscopy (i.e. longer illumination time) is a powerful technique for elucidating not only kinetics but also the multi-redox mechanisms at the electrode | electrolyte interface [6,33]. Special care must be taken regarding the chemical environment and wavelength used to excite the photoelectrode. TAS requires very specialised hardware and so it has not been implemented extensively for photoelectrode characterisation. 
(a)

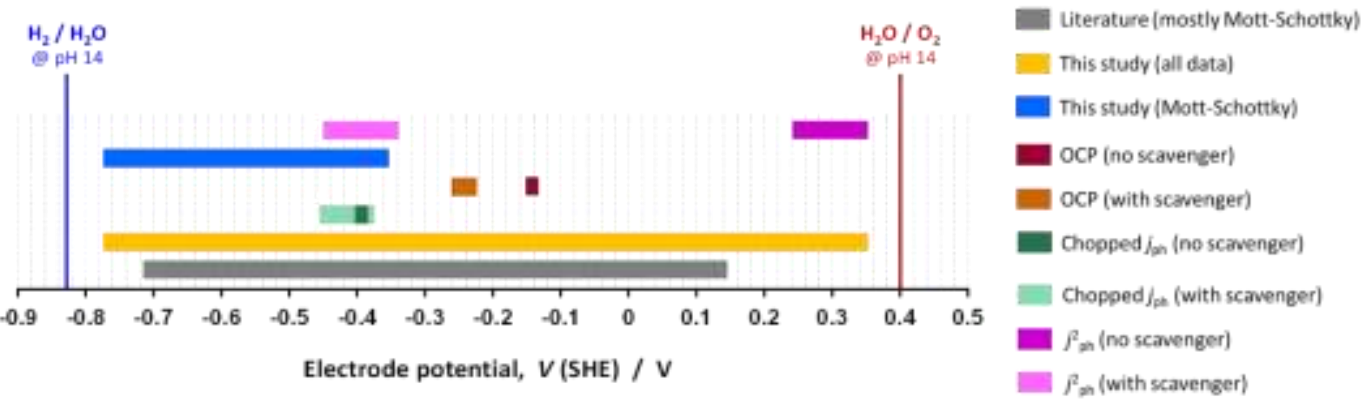

(b)

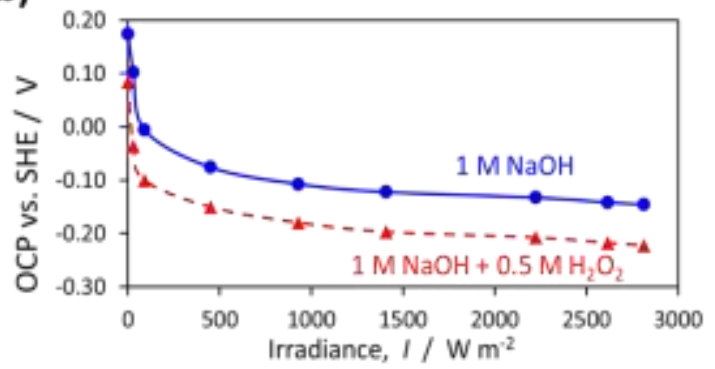

(d)

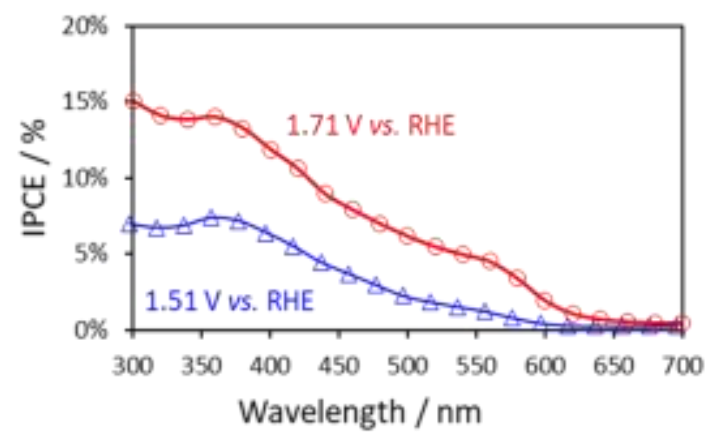

(f)

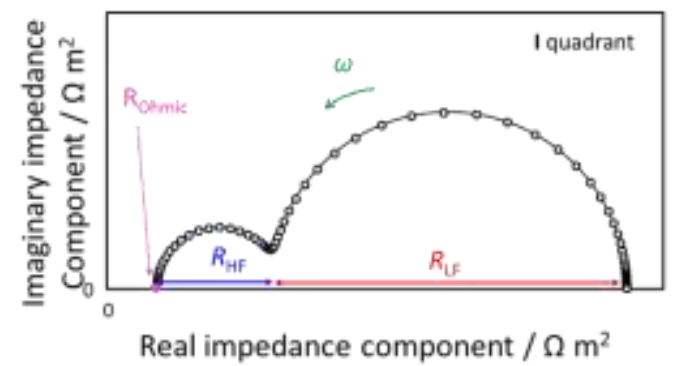

(c)

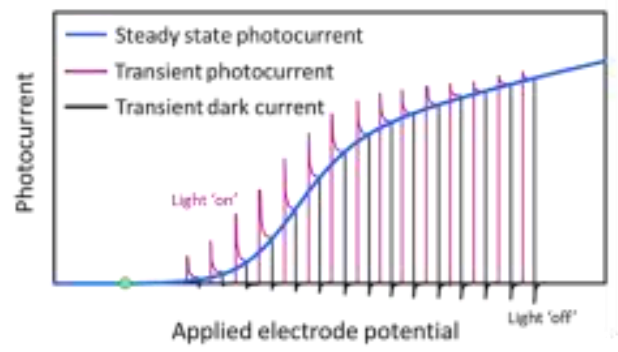

(e)

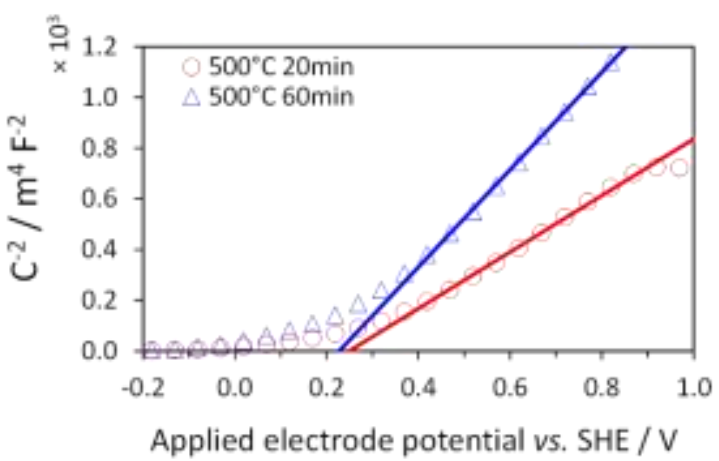

(g)

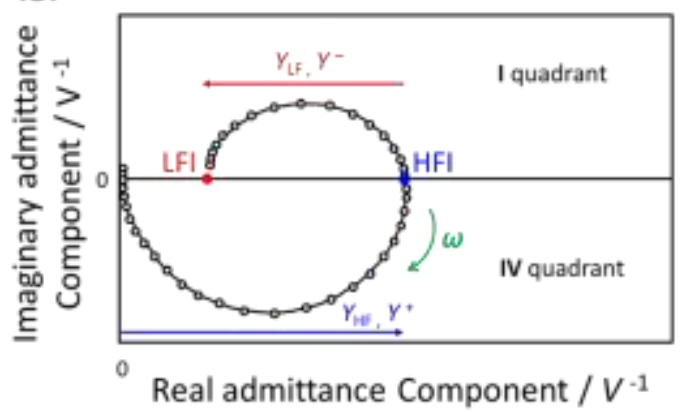

Figure 2. (a) Results map for flat band potentials determined using four experimental techniques: Mott-Schottky analysis of EIS data, OCP measurements, photocurrent squared and chopped photocurrent, on synthetic hematite in $1 \mathrm{M} \mathrm{NaOH}$ (with addition of $0.5 \mathrm{M} \mathrm{H}_{2} \mathrm{O}_{2}$ as hole scavenger for certain measurements) reported in [14] and tabulated values from an extensive literature review in [2]; (b) OCP for a hematite sample at different light intensities and electrolytes [14]; (c) Chopped photocurrent voltammetry for a typical photoanode; (d) IPCEs 
determined for hematite photoanodes under potentiostatic conditions; (e) Mott-Schottky plot from semiconductor capacitance values determined by electrical circuit fitting to EIS data [34]; (f) schematic of typical photo-impedance spectra exhibiting two processes at high (HF) and low (LF) frequencies and their respective resistances $\left(R_{\mathrm{HF}}\right.$ and $R_{\mathrm{LF}}$ ); (h) schematic of a typical IMPS spectra with the same two processes at high and low frequencies.

\section{Device metrics}

\subsection{Solar to hydrogen conversion efficiency (STH)}

The STH parameter is only appropriate if the device is capable of spontaneous water splitting without an external electrical power source and when the only energy output is the chemical product $\left(\mathrm{H}_{2}\right)$. More complex efficiency definitions are required if these conditions are not met [35].

A 'three-probe' half-cell electrochemical characterisation of the photoelectrode cannot be used to directly calculate the device STH, but could be predicted with caution utilising detailed device modelling [36-40]. That is, a photoanode that is characterized at $1.23 \mathrm{~V}$ (RHE) does not represent the photocurrent of a complete reactor as this neglects the operating condition dependent activation, concentration and ohmic losses, as well as product cross-over.

A $1^{\text {st }}$ law of thermodynamics type energetic conversion efficiency is defined as 'power converted' divided by the 'total input power'. The STH is commonly defined in Equation (3) in terms of the lower heating value $\left(237 \mathrm{~kJ} \mathrm{~mol}^{-1}\right.$ at $25^{\circ} \mathrm{C}$, resulting in $\left.\Delta V^{\varnothing}=1.23 \mathrm{~V}[41,42]\right)$ in contrast to typical electrolyser efficiency calculations, which use the thermo-neutral potential difference of $1.48 \mathrm{~V}$ $[43,44]$. It is equivalently calculated from the hydrogen production rate, which is superior as it implicitly includes the efficiency of gas collection as well as any losses due to product cross-over, or the electrical current flowing through the PEC device during spontaneous water splitting [45].

$$
\mathrm{STH}=\left[\frac{E_{\mathrm{H}_{2}} \cdot \dot{n}_{\mathrm{H}_{2}}}{P_{0} \cdot A}\right]_{\mathrm{AM} 1.5 \mathrm{G}}=\left[\frac{j_{\mathrm{ph}} \cdot \Delta V^{\varnothing} \cdot \varepsilon_{\mathrm{H}_{2}}}{P_{0}}\right]_{\mathrm{AM} 1.5 \mathrm{G}}
$$




\subsection{Solar to hydrogen conversion efficiency with externally applied bias}

The IUPAC-recommended modification to the STH calculation in the case of an externally applied bias is called the 'applied bias photon-to-current efficiency' (ABPE) and is shown in Equation (4) [45]. This parameter has been described as 'a diagnostic measurement in materials development' and cannot be considered a true energetic efficiency [45]. In addition to the pitfalls explained by multiple authors [35,45], the biggest drawback is that negative values for $\Delta V_{\text {cell }}>1.23 \mathrm{~V}$ are obtained, resulting in increasingly negative efficiencies for increasingly high photocurrents. This issue, illustrated in Figure 3(a), invalidates the equation for the very condition it is designed to represent. It is also noticeable that recent reviews and systematic studies of photoelectrode materials in biased systems do not use ABPE as an effective way to compare their performances [46-51] and it has been used sparsely and cautiously in recent studies [52-54]. A more rigorous formulation includes a sum of the power supplied by solar photons and via an applied bias in the denominator, giving a photo-assisted electrolysis system efficiency $\left(\eta_{\mathrm{PAE}}\right)$ in Equation (5); the results are shown in Figure 3(b) [35,38].

$$
\begin{gathered}
\mathrm{ABPE}=\left[\frac{j_{\mathrm{ph}} \cdot\left(\Delta V^{\varnothing}-\left|\Delta V_{\text {cell }}\right|\right)}{P_{0}}\right]_{\mathrm{AM} \mathrm{1.5G}} \\
\eta_{\mathrm{PAE}}=\left[\frac{E_{\mathrm{H}_{2}} \cdot \dot{n}_{\mathrm{H}_{2}}}{P_{0} \cdot A_{\text {photoabsorber }}+j_{\mathrm{ph}} \cdot \Delta V_{\text {cell }} \cdot A_{\text {electrode }}}\right]_{\mathrm{AM} \mathrm{1.5G}}
\end{gathered}
$$
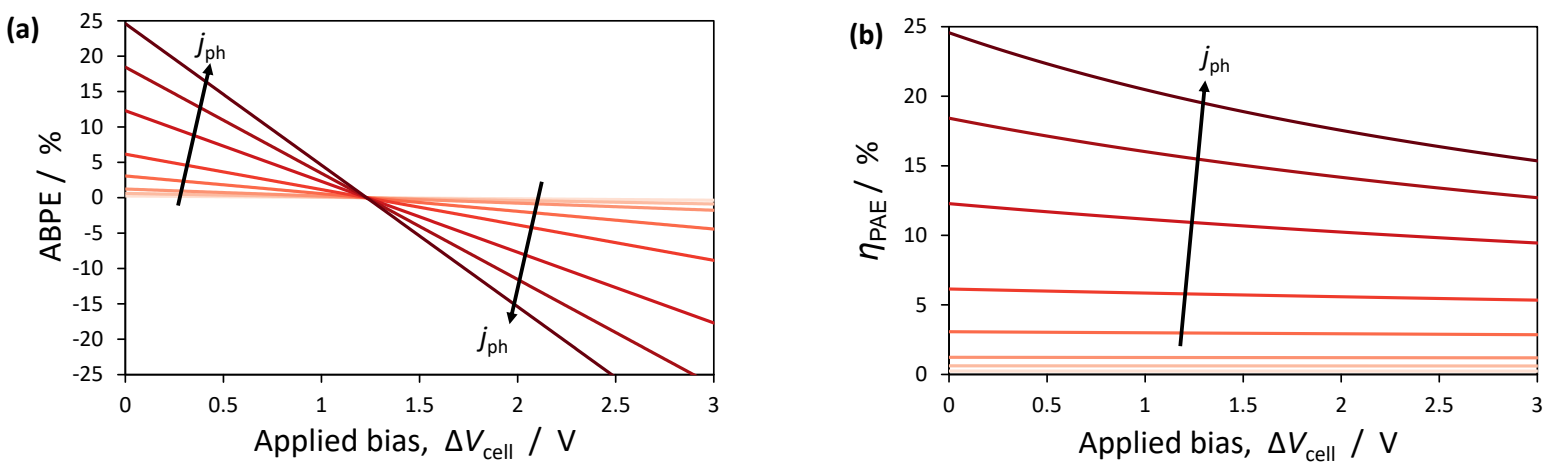

$$
j_{\mathrm{ph}}\left[\mathrm{mA} \mathrm{cm}^{-2}\right]: \quad \begin{array}{lllllllll}
0.2 & -0.5 & -1 & -2.5 & -5 & -10 & -15 & -20
\end{array}
$$


Figure 3. The dependence of the photocurrent density $\left(j_{\mathrm{ph}}\right)$ isolines on the externally applied cell bias ( $\left.\Delta V_{\text {cell }}\right)$ for (a) ABPE calculated using Equation (4) and (b) $\eta_{\mathrm{PAE}}$ calculated using Equation (5). $P_{0}$ fixed at $100 \mathrm{~mW} \mathrm{~cm}{ }^{-2}$. We note that this is for the purposes of investigating the behaviour of each equation and that for a typical device, the current density will change with applied bias.

\subsection{Outlook and perspectives}

We conclude that multiple methods should be employed to enable greatest confidence in experimentally determined parameters, such as the flat band potential and charge transfer efficiency. Apart from the newly developed techniques for locally resolved electrochemical characterisation, there is encouraging progress in complex models and postprocessing methods for impedance analysis, allowing researchers to circumvent traditional, although often controversial, a priori models. The practice of publishing open datasets from the aforementioned electrochemical characterisation of photo-electrodes (e.g. current density vs. voltage) should be mandated in order to permit the reproduction and comparison of experimental results and ensure the research integrity. There have been various attempts for unified benchmarking and characterisation

protocols; unfortunately, the photoelectrochemical community still lacks coherent procedures analogous to those used in photovoltaic characterisation, especially when it comes to stability and efficiency measurements and reporting. Due to the complex nature and multiple degradation mechanisms, descriptive statements such as "no morphology change was observed" or single performance metrics such as "5 \% decrease was measured after 24 hours" should always be accompanied by the supporting evidence, for example long-term chronoamperometry experiments or photographs/SEM images of electrodes before and after the test. If such raw data is absent, the validity of performance claims and replicability are severely negatively impacted. Comprehensive reporting guidelines and protocols should be the subject of a separate study.

The electrochemical characterisation of photoelectrodes arguably remains the most important set of analytical tools for assessing photoelectrode performance, and therefore the migration towards more unified and well-documented approach will facilitate the scaling up of these promising technologies. 


\subsection{Nomenclature}

Table 1 Terms and definitions used in this manuscript

$\begin{array}{ll}\text { Symbol or abbreviation } & \text { Definition } \\ \text { IPCE } & \text { Incident-to-photocurrent efficiency } \\ \text { RE } & \text { Reference } \\ \text { FB } & \text { Flat band } \\ \text { PEIS } & \text { Photoelectrochemical impedance spectroscopy } \\ \text { STH } & \text { Solar-to-hydrogen efficiency } \\ \text { ABPE } & \text { Applied bias photon-to-current efficiency } \\ \eta_{\mathrm{PAE}} & \text { Photo-assisted electrolysis system efficiency } \\ A & \text { Area } \\ C_{\mathrm{SC}} & \text { Semiconductor capacitance } \\ c, h, e & \text { Speed of light, Planck's constant, electronic charge } \\ E_{\mathrm{H}} & \text { Energy per mole of hydrogen } \\ \varepsilon_{0}, \varepsilon_{\mathrm{r}} & \text { Permittivity of free space, relative permittivity } \\ \varepsilon_{\mathrm{H}_{2}} & \text { Faradaic efficiency of } \mathrm{H}_{2} \text { evolution } \\ j_{\mathrm{ph}} & \text { Photocurrent density } \\ \lambda & \text { Photon wavelength } \\ k_{\mathrm{B}} & \text { Boltzmann constant } \\ n & \text { Dopant concentration (donor) } \\ \dot{n}_{\mathrm{H}_{2}} & \text { Molar flow rate of } \mathrm{H}_{2} \\ P_{0} & \text { Incident photon power density } \\ T & \text { Temperature } \\ \Delta V^{\varnothing} & \text { Water-splitting potential (typically 1.23 V) } \\ \Delta V_{\text {cell }} & \text { Applied cell bias } \\ V(\mathrm{RE}) & \text { Electrode potential relative to a reference electrode (RE) } \\ V_{\mathrm{FB}}(\mathrm{RE}) & \text { Flat band potential relative to a reference electrode (RE) } \\ & \end{array}$

\section{Acknowledgements}

The authors acknowledge the support of Colombia COLCIENCIAS scholarship 568, supporting a PhD studentship for F.B. and the UK EPSRC for post-doctoral research associateship for A.H., during which the opinions contained in the manuscript were formed. Special thanks to Dr. Camilo Mesa for his helpful feedback and suggestions.

\section{References}


[1] Y. Xu, M.A.A. Schoonen, The absolute energy positions of conduction and valence bands of selected semiconducting minerals, Am Mineral. 85 (2000) 543-556. https://doi.org/10.2138/am2000-0416.

[2] A. Hankin, J.C. Alexander, G.H. Kelsall, Constraints to the flat band potential of hematite photo-electrodes, Phys Chem Chem Phys. 16 (2014) 16176-16186. https://doi.org/10.1039/c4cp00096j.

[3] Yu.V. Pleskov, Photoelectrochemical Determination of the Flatband Potential of BoronDoped Diamond, Electrochem Solid-State Lett. 3 (1999) 141. https://doi.org/10.1149/1.1390982.

[4] A. Thakur, D. Ghosh, P. Devi, K.-H. Kim, P. Kumar, Current progress and challenges in photoelectrode materials for the production of hydrogen, Chemical Engineering Journal. 397 (2020) 125415. http://www.sciencedirect.com/science/article/pii/S1385894720314078.

[5] P. Tang, J. Arbiol, Engineering surface states of hematite based photoanodes for boosting photoelectrochemical water splitting, Nanoscale Horizons. 4 (2019) 1256-1276. https://doi.org/10.1039/c9nh00368a.

[6] C.A. Mesa, L. Francàs, K.R. Yang, P. Garrido-Barros, E. Pastor, Y. Ma, A. Kafizas, T.E. Rosser, M.T. Mayer, E. Reisner, M. Grätzel, V.S. Batista, J.R. Durrant, Multihole water oxidation catalysis on haematite photoanodes revealed by operando spectroelectrochemistry and DFT, Nat Chem. 12 (2020) 82-89. https://doi.org/10.1038/s41557-019-0347-1. 
[7] H. Wang, Y. Xia, H. Li, X. Wang, Y. Yu, X. Jiao, D. Chen, Highly active deficient ternary sulfide photoanode for photoelectrochemical water splitting, Nat Commun. 11 (2020) 3078. https://doi.org/10.1038/s41467-020-16800-w.

[8] F.E. Bedoya-Lora, A. Hankin, G.H. Kelsall, Hydrogen sulfide splitting using solar energy and hematite photo-anodes, Electrochimica Acta. 317 (2019) 384-397. http://www.sciencedirect.com/science/article/pii/S0013468619309417.

[9] E. Kalamaras, P. Lianos, Current Doubling effect revisited: Current multiplication in a PhotoFuelCell, Journal of Electroanalytical Chemistry. 751 (2015) 37-42. http://www.sciencedirect.com/science/article/pii/S1572665715002660.

[10] F. Nandjou, S. Haussener, Kinetic Competition between Water-Splitting and Photocorrosion Reactions in Photoelectrochemical Devices, Chemsuschem. 12 (2019) 1984-1994. https://doi.org/10.1002/cssc.201802558.

[11] I. Holmes-Gentle, F. Bedoya-Lora, F. Alhersh, K. Hellgardt, Optical Losses at Gas Evolving Photoelectrodes: Implications for Photoelectrochemical Water Splitting, J Phys Chem C. 123 (2018) 17-28. https://doi.org/10.1021/acs.jpcc.8b07732.

[12] Y. He, T. Hamann, D. Wang, Thin film photoelectrodes for solar water splitting, Chem Soc Rev. 48 (2019) 2182-2215. https://doi.org/10.1039/c8cs00868j.

[13] W. Yang, R.R. Prabhakar, J. Tan, S.D. Tilley, J. Moon, Strategies for enhancing the photocurrent, photovoltage, and stability of photoelectrodes for photoelectrochemical water splitting, Chem Soc Rev. 48 (2019) 4979-5015. https://doi.org/10.1039/c8cs00997j. 
*[14] A. Hankin, F.E. Bedoya-Lora, J.C. Alexander, A. Regoutz, G.H. Kelsall, Flat band potential determination: avoiding the pitfalls, J Mater Chem A. 7 (2019) 26162-26176. https://doi.org/10.1039/c9ta09569a. This study re-examined the methodologies used for flat band potential determination to help avoid misleading results from the Mott-Schottky approximation. The study demonstrated to the community why there is such a huge disparity in the flat band potential values reported in the literature and provided recommendations for how to improve the accuracy of reported values.

* [15] J. Zhang, S. Eslava, Understanding charge transfer, defects and surface states at hematite photoanodes, Sustain Energy Fuels. 3 (2019) 1351-1364. https://doi.org/10.1039/c9se00145j. This paper summarized and described several common issues with the popular hematite photoanodes by a thorough compilation of characterisation techniques and how these can be used to improve photoelectrode performances.

[16] Z. Zhou, S. Wu, C. Xiao, L. Li, W. Shao, H. Ding, L. Wen, X. Li, Self-improvement of solar water oxidation for the continuously-irradiated hematite photoanode, Dalton T. 48 (2019) 15151-15159. https://doi.org/10.1039/c9dt03368h.

[17] P. Córdoba-Torres, T.J. Mesquita, R.P. Nogueira, Relationship between the Origin of Constant-Phase Element Behavior in Electrochemical Impedance Spectroscopy and Electrode Surface Structure, J Phys Chem C. 119 (2015) 4136-4147. https://doi.org/10.1021/jp512063f.

[18] M.N. Kakaei, J. Neshati, A.R. Rezaierod, On the Extraction of the Effective Capacitance from Constant Phase Element Parameters, Prot Met Phys Chem S. 54 (2018) 548-556. https://doi.org/10.1134/s2070205118030280. 
[19] B. Hirschorn, M.E. Orazem, B. Tribollet, V. Vivier, I. Frateur, M. Musiani, Determination of effective capacitance and film thickness from constant-phase-element parameters, Electrochimica Acta. 55 (2010) 6218-6227.

http://www.sciencedirect.com/science/article/pii/S0013468609013413.

* [20] M.A. Danzer, Generalized Distribution of Relaxation Times Analysis for the Characterization of Impedance Spectra, Batter. 5 (2019) 53.

https://doi.org/10.3390/batteries5030053. This paper provides extremely valuable guidelines for carrying out reliable and reproducible DRT analysis, which is a relatively new technique for processing impedance data.

[21] D. Klotz, D.A. Grave, A. Rothschild, Accurate determination of the charge transfer efficiency of photoanodes for solar water splitting, Phys Chem Chem Phys. 19 (2017) 2038320392. https://doi.org/10.1039/c7cp02419c.

[22] J. Liu, F. Ciucci, The Gaussian process distribution of relaxation times: A machine learning tool for the analysis and prediction of electrochemical impedance spectroscopy data, Electrochimica Acta. 331 (2020) 135316. http://www.sciencedirect.com/science/article/pii/S0013468619321887.

[23] X. Huai, L. Girardi, R. Lu, S. Gao, Y. Zhao, Y. Ling, G.A. Rizzi, G. Granozzi, Z. Zhang, The mechanism of concentric $\mathrm{HfO} 2 / \mathrm{Co} 3 \mathrm{O} 4 / \mathrm{TiO} 2$ nanotubes investigated by intensity modulated photocurrent spectroscopy (IMPS) and electrochemical impedance spectroscopy (EIS) for photoelectrochemical activity, Nano Energy. 65 (2019) 104020.

https://doi.org/10.1016/j.nanoen.2019.104020. 
* [24] S. Ravishankar, A. Riquelme, S.K. Sarkar, M. Garcia-Batlle, G. Garcia-Belmonte, J. Bisquert, Intensity-Modulated Photocurrent Spectroscopy and Its Application to Perovskite Solar

Cells, J Phys Chem C. 123 (2019) 24995-25014. https://doi.org/10.1021/acs.jpcc.9b07434. This study presents an authoritative review of the fundamentals of intensity-modulated photocurrent spectroscopy and a critical appraisal of equivalent circuit modelling. Whilst this study focuses mainly on perovskite solar cells, it is instructive for all IMPS users.

[25] M. Antuch, P. Millet, A. Iwase, A. Kudo, The role of surface states during photocurrent switching: Intensity modulated photocurrent spectroscopy analysis of BiVO4 photoelectrodes, Applied Catalysis B: Environmental. 237 (2018) 401-408.

http://www.sciencedirect.com/science/article/pii/S0926337318304296.

[26] D. Klotz, Negative capacitance or inductive loop? - A general assessment of a common low frequency impedance feature, Electrochemistry Communications. 98 (2019) 58-62. http://www.sciencedirect.com/science/article/pii/S1388248118303084.

[27] L.M. Peter, Energetics and kinetics of light-driven oxygen evolution at semiconductor electrodes: the example of hematite, J Solid State Electr. 17 (2013) 315-326. https://doi.org/10.1007/s10008-012-1957-3.

[28] C.Y. Cummings, F. Marken, L.M. Peter, A.A. Tahir, K.G.U. Wijayantha, Kinetics and mechanism of light-driven oxygen evolution at thin film $\alpha$-Fe 2 O 3 electrodes, Chem Commun. 48 (2011) 2027-2029. https://doi.org/10.1039/c2cc16382a.

[29] A. Kafizas, X. Wang, S.R. Pendlebury, P. Barnes, M. Ling, C. Sotelo-Vazquez, R. QuesadaCabrera, C. Li, I.P. Parkin, J.R. Durrant, Where Do Photogenerated Holes Go in Anatase:Rutile 19 
TiO2? A Transient Absorption Spectroscopy Study of Charge Transfer and Lifetime, J Phys Chem. 120 (2016) 715-723. https://doi.org/10.1021/acs.jpca.5b11567.

* [30] A. Kafizas, X. Xing, S. Selim, C.A. Mesa, Y. Ma, C. Burgess, M.A. McLachlan, J.R. Durrant, Ultra-thin A12O3 coatings on BiVO4 photoanodes: Impact on performance and charge carrier dynamics, Catalysis Today. 321-322 (2019) 59-66.

http://www.sciencedirect.com/science/article/pii/S0920586117307666. This study used advanced characterisation techniques to unravel the charge carrier dynamics in a protected photoelectrode. It used a wide range of well described and documented techniques with a focus on transient absorption spectroscopy (TAS).

[31] S. Selim, E. Pastor, M. García-Tecedor, M.R. Morris, L. Francàs, M. Sachs, B. Moss, S. Corby, C.A. Mesa, S. Gimenez, A. Kafizas, A.A. Bakulin, J.R. Durrant, Impact of Oxygen Vacancy Occupancy on Charge Carrier Dynamics in BiVO4 Photoanodes, J Am Chem Soc. 141 (2019) 18791-18798. https://doi.org/10.1021/jacs.9b09056.

[32] M. Sachs, E. Pastor, A. Kafizas, J.R. Durrant, Evaluation of Surface State Mediated Charge Recombination in Anatase and Rutile TiO2, J Phys Chem Lett. 7 (2016) 3742-3746. https://doi.org/10.1021/acs.jpclett.6b01501.

[33] A. Kafizas, Y. Ma, E. Pastor, S.R. Pendlebury, C. Mesa, L. Francàs, F.L. Formal, N. Noor, M. Ling, C. Sotelo-Vazquez, C.J. Carmalt, I.P. Parkin, J.R. Durrant, Water Oxidation Kinetics of Accumulated Holes on the Surface of a TiO2 Photoanode: A Rate Law Analysis, Acs Catal. 7 (2017) 4896-4903. https://doi.org/10.1021/acscatal.7b01150. 
[34] F.E. Bedoya-Lora, A. Hankin, I. Holmes-Gentle, A. Regoutz, M. Nania, D.J. Payne, J.T. Cabral, G.H. Kelsall, Effects of low temperature annealing on the photo-electrochemical performance of tin-doped hematite photo-anodes, Electrochimica Acta. 251 (2017) 1-11. http://www.sciencedirect.com/science/article/pii/S0013468617317310.

[35] R.H. Coridan, A.C. Nielander, S.A. Francis, M.T. McDowell, V. Dix, S.M. Chatman, N.S. Lewis, Methods for comparing the performance of energy-conversion systems for use in solar fuels and solar electricity generation, Energ Environ Sci. 8 (2015) 2886-2901. https://doi.org/10.1039/c5ee00777a.

[36] I. Holmes-Gentle, F. Hoffmann, C.A. Mesa, K. Hellgardt, Membrane-less photoelectrochemical cells: product separation by hydrodynamic control, Sustain Energy Fuels. 1 (2017) 1184-1198. https://doi.org/10.1039/c7se00176b.

[37] F.E. Bedoya-Lora, A. Hankin, G.H. Kelsall, En route to a unified model for photoelectrochemical reactor optimisation. I - Photocurrent and H2 yield predictions, J Mater Chem A. 5 (2017) 22683-22696. https://doi.org/10.1039/c7ta05125e.

[38] A. Hankin, F.E. Bedoya-Lora, C.K. Ong, J.C. Alexander, F. Petter, G.H. Kelsall, From millimetres to metres: the critical role of current density distributions in photo-electrochemical reactor design, Energ Environ Sci. 10 (2017) 346-360. https://doi.org/10.1039/c6ee03036j.

* [39] M.R. Singh, S. Haussener, A.Z. Weber, Chapter 13 Continuum-scale Modeling of Solar Water-splitting Devices, in: The Royal Society of Chemistry, 2019: pp. 500-536.

https://doi.org/10.1039/9781788010313-00500. This comprehensive review of modelling solar 
fuel devices has an emphasis on multi-physical, multi-dimensional modelling and notably presents the governing equations for each of the relevant physical phenomena.

[40] M. Lin, J. Reinhold, N. Monnerie, S. Haussener, Modeling and design guidelines for direct steam generation solar receivers, Applied Energy. 216 (2018) 761-776.

http://www.sciencedirect.com/science/article/pii/S0306261918301697.

[41] O. Khaselev, A. Bansal, J.A. Turner, High-efficiency integrated multijunction photovoltaic/electrolysis systems for hydrogen production, International Journal of Hydrogen Energy. 26 (2001) 127-132. https://doi.org/10.1016/s0360-3199(00)00039-2.

[42] C. Carver, Z. Ulissi, C.K. Ong, S. Dennison, G.H. Kelsall, K. Hellgardt, Modelling and development of photoelectrochemical reactor for $\mathrm{H} 2$ production, Int J Hydrogen Energ. 37 (2012) 2911-2923. https://doi.org/10.1016/j.ijhydene.2011.07.012.

[43] K.W. Harrison, R. Remick, G.D. Martin, A. Hoskin, Hydrogen Production: Fundamentals and Cas Study Summaries, in: D. Stolten, T. Grube (Eds.), 18th World Hydrogen Energy Conference 2010 - WHEC 2010, 2010. https://juser.fzjuelich.de/record/135438/files/HP3a_1_Harrison_10.pdf.

[44] R. Phillips, W.J.F. Gannon, C.W. Dunnill, Alkaline Electrolysers, in: K. Scott (Ed.), Electrochemical Methods for Hydrogen Production, The Royal Society of Chemistry, 2019: pp. 28-58. https://doi.org/10.1039/9781788016049.

[45] Z. Chen, T.F. Jaramillo, T.G. Deutsch, A. Kleiman-Shwarsctein, A.J. Forman, N. Gaillard, R. Garland, K. Takanabe, C. Heske, M. Sunkara, E.W. McFarland, K. Domen, E.L. Miller, J.A. 
Turner, H.N. Dinh, Accelerating materials development for photoelectrochemical hydrogen production: Standards for methods, definitions, and reporting protocols, J Mater Res. 25 (2010) 3-16. https://doi.org/10.1557/jmr.2010.0020.

[46] K. Sivula, F. Le Formal, M. Grätzel, Solar Water Splitting: Progress Using Hematite ( $\alpha$ Fe2O3) Photoelectrodes, Chemsuschem. 4 (2011) 432-449.

https://doi.org/10.1002/cssc.201000416.

[47] B. Iandolo, B. Wickman, I. Zorić, A. Hellman, The rise of hematite: origin and strategies to reduce the high onset potential for the oxygen evolution reaction, J Mater Chem A. 3 (2015) 16896-16912. https://doi.org/10.1039/c5ta03362d.

[48] C. Acar, I. Dincer, A review and evaluation of photoelectrode coating materials and methods for photoelectrochemical hydrogen production, Int J Hydrogen Energ. 41 (2016) 79507959. https://doi.org/10.1016/j.ijhydene.2015.11.160.

[49] P. Dias, A. Vilanova, T. Lopes, L. Andrade, A. Mendes, Extremely stable bare hematite photoanode for solar water splitting, Nano Energy. 23 (2016) 70-79. https://doi.org/10.1016/j.nanoen.2016.03.008.

[50] X. Chen, Z. Zhang, L. Chi, A.K. Nair, W. Shangguan, Z. Jiang, Recent Advances in Visible-Light-Driven Photoelectrochemical Water Splitting: Catalyst Nanostructures and Reaction Systems, Nano-Micro Lett. 8 (2016) 1-12. https://doi.org/10.1007/s40820-015-0063-3. 
[51] Y. Ling, Y. Li, Review of Sn-Doped Hematite Nanostructures for Photoelectrochemical Water Splitting, Part Part Syst Char. 31 (2014) 1113-1121. https://doi.org/10.1002/ppsc.201400051.

[52] H. Lim, J.L. Young, J.F. Geisz, D.J. Friedman, T.G. Deutsch, J. Yoon, High performance III-V photoelectrodes for solar water splitting via synergistically tailored structure and stoichiometry, Nat Commun. 10 (2019) 3388. https://doi.org/10.1038/s41467-019-11351-1.

[53] K.-H. Ye, H. Li, D. Huang, S. Xiao, W. Qiu, M. Li, Y. Hu, W. Mai, H. Ji, S. Yang, Enhancing photoelectrochemical water splitting by combining work function tuning and heterojunction engineering, Nat Commun. 10 (2019) 3687. https://doi.org/10.1038/s41467-01911586-y.

[54] J. Hu, S. Zhao, X. Zhao, Z. Chen, Strategies of anode materials design towards improved photoelectrochemical water splitting efficiency, Coatings. 9 (2019) 309. 